\title{
Die gefässlose Stelle der mensohliohen Retina und deren Verwerthung zur Bestimmung der Ausdehnung dor Macula lutea.
}

Von

Dr. Demetrius P. Johannides, II. Assistenten am anatomischen Institut in Erlangen.

(Hierzu Tafel IV).

Die Gefăssverhältnisse des menschlichen Auges sind durch zahlreiche Arbeiten vieler tüchtiger Forscher ziemlich genan bekannt. Allein die meisten Gefässuntersuchungen, auch durch treffliche Abbildungen erläntert, beziehen sich auf die äusseren Augenhäute, während die Gefässverhältnisse der Retina nur nebenbei berücksichtigt wurden, insofern das Gefässsystem des Nervus opticus und der Retina mit dem Ciliargefässsystem in Zusammenbang steht. Dieser Zusammenhang, dessen Kenntniss wir Th. Leber*) verdanken, ist ein doppelter, nänlich in der Höhe des Durchtritts des Nervus opticus

*) Anatomische Untersuchungen über die Blutgefässe des menschlichen Auges, Denkschriften der $\mathrm{k}$. Academie der Wissensch. zu Wien. Math.-naturwiss. Class. Bd, 24. S. 297-330. - GraefeSämisch. Handbuch der gesammten Augenheilkunde. Bd. 2. S. 302. 1876. 
durch die Sclera ein indirecter, durch den von Zinn entdeckten Scleralgefässkranz (Circulus arteriosus nervi optici) vermittelt; und dann ein directer, indem in der unmittelbaren Nähe der Papilla N. optici sowohl das Capillarnetz der Chorioidea wie die grösseren Gefasse derselben*) mit den Gefässen des Nervus opticus anastomosiren.

Der Grund, weshalb das eigentliche Gefässnetz der Retina im Ganzen bisher wenig berücksichtigt wurde, scheint ein doppelter zu sein. Einmal konnte vor der Einführung durchsichtiger Injectionsmassen, namentlich der am leichtesten vordringenden GelatinCarmin-Ammoniummasse, bei der Feinheit der Capillaren der Retina und der zartesten Structur dieser Membran von fehlerfreien Injectionen überhaupt nicht die Rede sein. Der andere Grund liegt in der Schwierigkeit sich frische menschliche Augen von Kindern zu verschaffen, welche allein günstige Injectionserfolge versprechen. Substituirung von Thieraugen ist aber hier ganz unstatthaft, da die Gefăssverhältnisse auch der Säugethierretina von jenen des Menschen ganz wesentlich abweichen. Ganz abgesehen davon, dass mit Ausnahme des Affen den Augen der Säugethiere die Macula lutea fehlt, welche wegen ihrer Gefässlosigkeit gerade für die Gefässverhältnisse der menschlichen Retina der interessanteste Theil ist, erscheint bei vielen Säugern die Retina nur ganz partiell mit Gefässen versehen, so bei Kaninchen, Meerschweinchen, Pferden. Die gefässärmste Retina scheint die der Gürtelthiere zu sein.

*) Ich erlaube mir hierzu zu bemerken, dass ich mich nicht bestimmt davon überzeugen konnte, ob die Gefässe von mehr als capillarem Kaliber, welche vom Aderhautrande aus in den Sehnerven übertreten, direct mit den entsprechenden Gefässen des letzteren anastomosiren, oder ob sie damit nur durch das Capillar netz in Verbindung sttehen. (Vergl. dieses Archiv XVII, 2. S. 31-32). Th. Leber. 
Die beiden hauptsächlichen Thatsachen, welche bisher über die Gefässverbältnisse der Retina bekannt waren, sind: erstens die Gefässlosigkeit der Fovea centralis und zweitens die Beobachtung, dass die grösseren Gefässe der Retina sämmtlich in der Faserschicht dieser Membran verlaufen, während die eigentlichen Capillarmaschen sich nicht weiter nach aussen als bis zur Zwischenkörnerschicht erstrecken, so dass nur die nervöse Lage der Retina gefässhaltig, die percipirenden oder musivisehen Schichten derselben vollständig gefässlos sind.

In der vorliegenden Arbeit beabsichtige ich eine Darstellung der Gefässe der Retina in der Umgebung der Macula lutea zu geben, namentlich zu bestimmen, wie sich der gefässhaltige Theil der Retina von dem nicht gefässhaltigen abgrenzt, und aus der râumlichen Ausdehnung des letzteren einen Schluss zu ziehen auf die Grösse der Macula lutea, die ja wegen des allmähligen Ueberganges der gefärbten Stelle in die nicht gefürbte auf eine andere Weise scharf nicht bestimmt werden kann.

Als Unterlage diente mir die injicirte Netzhant eines vierjährigen Kindes, welche von der Arteria ophthalmica aus mit Carmin-Ammoniummasse vollständig gefïllt worden war. Dieses Präparat, das aus der quantitativ wie qualitativ ausgezeichneten Sammlung des Herrn Prof. von Gerlach stammt, bringt eine evidente Entscheidung über die in der Gegend der Macula lutea in Wirklichkeit bestehenden Gefässverhältnisse, deren richtige Kenntniss nicht nur interessant für den Anatomen von Fach, sondern auch für den Ophthalmologen wichtig für die Deatung gewisser pathologischer Veränderungen an dieser Stelle des Augenhintergrundes ist.

Der Beschreibung des Präparates möge ein Blick auf die Literatur vorausgehen.

マ. Graefe's Arehiv für Ophthaimologie. XXVI. 2. 
Die ersten, welche auf die Gefässlosigkeit der Macula Iutea aufmerksam machten, waren Sömmèring*) und Purkinje**), der erstere auf rein anatomische Beobachtung sich stützend, der letztere von subjectiven physiologischen Versuchen ausgehend, indem er zeigte, dass es möglich ist, die Gefässe seiner Retina, Aderfigur ge* nannt, zu beobachten.

Sömmering äusserte sich folgendermassen:

„Arteriae enim of venae, per mediam nervi optici substantiam penetrantes, ita in Retina sparguntur, ut earum rami majores semper adeo remote ab hoc foraminulo decurrant, uf foraminulum ne attingere quidem nedum transire queant, sed aequali fere distantia superius et inferius ab illo foraminulo centrali ludentes rami ramuseulos versus ambitum hujus foraminuli ablegant quorum surculi subtilissimi tantum ad limbum perveniunt. Quare sertum quasi quoddum raseulare a duobus ramis majoribus vasorum sanguifororum cirenm illad foramen centrale limbo lnteo cinctum construitur, cujus racemuli subtilissimi tantum limbum ipsum attingunt."

Bei Betrachtung der der Sömmering'schen Abhandlung beigegebenen Tafel Fig. 1, 2 und 4 wirt alsbald klar, dass sich die Sömmerring'sche Beschreibung nicht auf die Capillaren, sonderu nur auf die gröberen Gefässe der Retina bezieht.

Purkinje wandte zur subjectiven Wahrnehmung der Aderfigur drei Methoden an:

1) Hart vor der Cornea wird, während der Blick gegen den Himmel gerichtet ist, ein undurchsichtiger Schirm mit feiner runder Oeffnngg hin und her bewegt.

*) De foramine centrali limbo luteo cincto retinae humanae. Commentationes societatis regiae scientiarum Gottingensis ad a. 17951798. val. XIII. p. 7. 1799.

**) Beobachtungen und Versuche zur Physiologie der Sinne. Bd. I. S. 89. Prag, 1828 und Bd, II, S, 117. Berlin, 1825. 
2) Eine Kerzenflamme wird nabe vor dem Auge langsam in verschiedenen Richtungen, wohl auch im Kreise berum geschoben.

3) Mittelst einer Convexlinse wird Licht auf die Sclera concentrirt, die Linse hin und her bewegt, während der Blick einem dunkeln Hintergrund zugewandt ist.

Auch durch Druck auf den äusseren oberen Quadranten des Augapfels will Purkinje die Aderfigur sichtbar gemacht haben. Derselbe gibt an, dass er die Gefässe der Retina bis zu den feinsten Verzweigungen auf diese Art verfolgen konnte, und sagt weiter, dass die auf diese Weise beobachteten Gefässe der Retina dasselbe Verhalten zeigen, wie die Abbildungen, die Sömmering zuerst über die Gefässe der Retina gegeben. Dabei bemerkt Purkinje ausdrücklich, dass ein kleiner Theil der Retina, in dem hinteren Pol der Sehaxe gelegen, also dem gelben Fleck entsprechend, vollkommen gefässlos bleibt.

Nach Entdeckung des Augenspiegels wurden die Gefässverhältnisse der Retina am Lebenden der directen Beobachtung zugänglich. Auffallender Weise haben die beiden Autoren, welche die genauesten Beschreibungen des ophthalmoscopischen Befundes der Macula lutea gaben, Schirmer und Delorme, die Gefässlosigkeit der Macula lutea in Abrede gestellt.

So sagt Schirmer*):

„Bei allen jugendlichen Individuen, mag bei ihnen das Chorioidealpigment kaum oder mässig oder stark entwickelt sein, erkenne ich stets die Macula lutea zuerst an einem sie umkreisenden, querovalen, hellglänzenden Reifen von der Dicke einer Retinalarterie erster Grösse. Der innere Rand dieses

*) Ueber das ophthalmoscopische Bild der Macula lutea. v. Graefe's Archiv für Ophthalmologie Bd X., Abth. 1., S. 148. 
Reifens ist scharf, der äussere aber mit kurzen, dichten und feinen Strahlen versehen, so dass die ganze Figur wie ein Heilig nschein aussieht. Wenn man die Papille im Gesichtsfelde hat, so erblickt man nicht den ganzen Reif $n$, sondern nur eine seitliche Hälfte desselben; in anderen Fallen hat man nur eine obere oder untere Halfte im Gesichtsfelde. Ausserhalb dieses Reifens sieht man den hellen rothen Augenhintergrund; er selbst aber schliesst eine dunklere matte Partie ein. Ich orkenno also hiernach den gelben Fleck nicht bloss als dunkle, maite Stelle in dem allgemeiren Glanz der Retina, nicht bloss als einen Defect in demselben, sondern ich seho stets einen circumseripten, glänzenden Ring um die Stelle des dentlichsten Sehens.

Der Glanz tritt hier gerade so auf, wie an einer Seite der Netzhautgefässe, die ja oft ron einem hellen Streifen berandet erscheinen; und zwar machte ich bei letzteren die Bemerkung, dass dieser Glanz an den dem Kernlicht des Augenspiegels abgewandten Seiten der Gefüsse sichtbar wird. Nur selten sieht man auf beiden Saiten der Adern zugleich, wenn dieselben ziemlich in der Witte des belenchteten Feldes liegen, diesen glänzenden Strejfen; das so rom Glanz eingeschlossene Gefäss wird dann streckenweise verdeckt und sieht so einer varicösen Vene âhnlich. Manchmal, besonders in der Nahe der Macula latea, bekommt man Partien zn Gesicht, deren Aussehon an kleine, feinverästelte, hellglänzende Eisblumen erinnert. Auch dies hat seinen Grund in der Gestaltung der feinsten Gefässausbreitungen.

Demgemäss liegt die Frage nahe, ob nicht auch durch die Retinalgefässe, welecte die Maenla lutea umkreisen, jener "Heiligenschein" erengt wird. Dies ist zu verneinen: einen solchen Gefässkranz fand ich daselbst zie, vieimehr sah ich mehrfache kleine Gefässe in die Macula eintreten, und zwar zumeist von oben und unten, ja sogar bis dieht an die Forea centralis hin. Der gelbe Fleck ist demnach keine gefasslose Stelle, wie er fast allgemein beschrieben wird."

Auch E. Delorme*), dessen ophthalmoseopische Darstellungen der Gefässe in der Gegend der Macula

*) Journal d'Ophthalmologie. Jahrg. 1872. S. 92. 
lutea für sehr gut gelten, behauptet, dass in der Macula lutea mehr kleine Gefässverzweigungen gesehen werden, als in irgend einem anderen Theile der Retina. In seiner ophthalmoscopischen Zeichnung sieht man aber diesen Reichthum an feinen Capillargefässen der Macula lutea nicht, wie ich überhaupt keine ophthalmoscopische Zeichnung gesehen habe, wo die Macula lutea mit ihren vermutheten Gefässen abgebildet wird. Das war freilich auch zu erwarten; denn der Augenspiegel vermag nur die gröberen, macroscopischen Gefässe der Retina zur Anschauung zu bringen, und um feinste Gefässverhältnisse derselben sichtbar zu machen ist er ein unzureichendes Mittel. Demnach müssen die gemachten Angaben und Behauptungen zurückgewiesen werden und können keinen Anspruch darauf machen, die rein anatomische Frage rücksichtlich der Gefässverhältnisse der Macula lutea zur Entscheidung za bringen.

Wenden wir uns jetzt den neuen Autoren zu, welche unter Anwendung der modernen Injectionsmethoden die Frage vom rein anatomischen Standpunkt aus behandelten. Die erste hierher gehörige Mittheilung finde ich bei J. Niemetschek*), welcher sagt:

„Bei gelungenen Injectionen der Netzhant findet man ein viel dichteres Capillarnetz, als man nach den ophthalmoseopisehen Befunden vermuthen würde. Selbst in der Maeula lutea, die gewöhnlich für gefässlos gilt, findet man bei Nengeborenen und Erwachsenen ein feines Capillarnetz. Die Gefăsse haben an dieser Netzhautstelle einen zum Foramen centrale tendirenden Verlauf. Vorwaltend entwickelt sind daselbst die von oben und unten kommenden Reiser, schwächer die von innen, am schwächsten die von aussen gegen die Mitte der Forea ziehenden Capillaren, Sie verflechten sich an der Hacula lutea zu einem Netze, dessen Masehen ziemlich weit und

*) Prager Vierteljahrschrift. Bd. I., S. 132. 1866. 
in der Regel gestreckt sind. Die Fovea-Gefasse sind hauptsächlich Zweige der um die Stelle des directen Sehens bogenförmig verlaufenden Aeste der Centralis retinae. Bei unvollkommenen Injectionen bleibt die gefärbte Nasso in der Regel an đer Grenze der Macula stehen und die Gefässe scheinen dort plotzlich zu endigen. Doch deuten schon die plötzlichen Endigungen darauf hin, dass die Masse nicht bis za den feinsten Verzweigungen rordrang."

Auch von E. Nettleship ${ }^{*}$ ), Wundarzt am ophthalmologischen Hospital zu Süd-London, haben wir eine Beschreibung der Blutgefässe der Retina aus der Gegend der Macula lutea, welche durch einen Holzschnitt illustrirt ist. Nettleship behauptet, dass die Fovea centralis der einzige Theil der Retina sei, welcher keine Blutgefässe und Capillaren enthalte. Er stützt sich dabei auf ein Präparat der menschlichen Retina, deren Gefässe mit der durch Essigsäure leicht angesäuerten CarminAmmoniummasse gefüllt worden waren. Die beigegebene Abbildung veranschaulicht nicht das, was der Autor behauptet. Es erscheint darin nur das die Macula lutea umgebende, dichtere Capillarnetz, während die Capillargefässe der Macula lutea selbst unsichtbar bleiben. Die Injection scheint unvollständig; denn einzelne Capillarschlingen sind zerrissen, andere verdreht und einzelne haben sich nicht gefüllt, wie Nettleship selbst zugibt.

Auch in den neueren Handbüchern der Histologie und Ophthalmologie ist durchgehends die Ansicht vertreten, dass nur die Fovea centralis gefássios sei.

So äussert sich Schwalbe**):

"Auf der Oberfläche der Papilla n. optici theilen sich Arteria und Vena centralis retinae in ïhre Hauptäste, die

*) Note on the retinal blood-vessels of the yollow-spotregion. Ophthalmic Hospital Reports and Journal of the Royal Iundon Ophthalmic Hospital, Vol. 8, Part. 2. 1875.

**) Graefe-Samiseh. Handbuch der gesammten Augenhellkande. Bd. I. S. 441. Berlin, 1874. 
Ven $\bullet$ meist früher als die Arterie. ... Die Theilungen erfolgen dichotomisch. Gerade nach aussen zur Macula lutea dringt kein grösserer $\mathrm{Zweig}$ der Arterie und Vene. Dieselbe wird vielmehr voun den Gefăssen im Bogen umzogen, wobei letztere von allen Seiten kleinere Gefässe in den gelben Fleck hinein senden, ebenso wie von der Papille direct einige feine Gefässe dorthin verlaufen. In der Macula lutea dringen Capillaren bis zum Rande der Fovea centralis vor, wo sie schlingenförmig umbiegen, so dass die Fovea selbst gefässlos bleibt.

Frey*) giebt an:

„dass in dem gelben Fleck zahlreiche Capillaren, aber keine stärkeren Gefässröhren auftreten."

E. Meyer**) bemerkt:

"Die Macula lutea wird von den gröberen Gefässen in Bogen umkreist, aus deren, dem gelben Fleck zugekehrter Concavität feine $Z$ weige in den letzteren eintreten, welche sich nach der Fovea centralis zu in Capillaren auflösen. Die Fovea centralis selbst wird ron diesem Capillarnetz nicht erreieht. Dieselbe ist rollkommen gefässlos."

Loring***) sagt:

„Während Andere die Macula lutea für fast gefässlos halten, ist diese Stelle vielmehr derjenige Theil der Retina, in welchem die meisten, aber auch die kleinsten Gefässverzweigungen vorkommen. - Aehnlich wie mit den Nervenfasern verbält es sich, nach Manz $\dagger$ ), auch mit den Blutgefässen; diese ziehen, die grösseren Aeste wenigstens, in ziemlich grossen Bogen um die Macula herum, während nur ihre feineren Zweige gegen sie herantreten, ohne aber die Fovea selbst zu erreichen."

*) Handbuch der Histologie und Histochemie des Menschen S. 669. Leipzig, 1874.

**) Handbuch der Augenheilkunde. S. 217. Berlin, 1875.

***) American Journal of Medical Sciences, April, 1874. S. 313-328.

†) Entwicklungsgeschichte des menschlichen Auges. GraefeSämiseh. Bd. 2. S. 48.1876. 
Auch Leber*) meint:

„dass die Macula lutea bloss von grösseren Gefässen gemieden wird, während feine $Z$ weige von den sie umkreisenden Gefässen in dieselbe sich erstrecken und bis an den Rand der Fovea reichen, wo sie dann mit einem Kranz von eapillaren Schlingen endigen, so dass die Fovea allein vollkommen gefässlos ist."

Die zu- und abführenden Blutgefâssse der Retina sind die Vasa centralia. Dieselben verlaufen in der Axe des optischen Nerven und zwar dringt die Arterie in der Regel $15 \mathrm{~mm}$, die Vene dagegen nur $10 \mathrm{~mm}$ von dem Augapfel entfernt in den Nervus opticus ein. In der Axe des optischen Nerven liegen die Gefässe neben einander bis zu der Papilla nervi optici, wobei sich die Vene etwas früher theilt als die Arterie und zwar zuerst in einen oberen und unteren Ast. Diese erste Theilung fällt noch in den Bereich der Papille; die zweite Theilung dagegen geschieht an dem Rande der Papille, von wo an vier Aeste bereits vorhanden sind, von denen der eine aufwärts, der andere abwärts, der dritte nasal und der vierte temporal verläuft. Die venösen Aeste sind nahe zu einem Dritttheile weiter als die arteriellen Aeste. Von den directen und indirecten Anastomosen der Retinagefässe mit dem Ciliargefässsystem an der Eintrittsstelle des Sehnerven in den Bulbus war bereits oben die Rede. Vor der Papilla nervi optici existirt keine Anastomose zwischen den beiden Gefässsystemen namentlich nicht an der Ora serrata der Retina. Die Gefässe der Retina erstrecken sich überhaupt nur bis an die Ora serrata; die Pars ciliaris ist absolut gefässlos. Die Arterien der Netzhaut anastomosiren niemals unter einander und auch Venenanastomosen kommen

*) Graefe-Sämisch. Bd. 2. s. 309 . 
nur zwischen kleinen Venen in der Nähe der Ora serrata vor. Die Venen treten etwas näher an die Ora serrata heran, als-die Arterien, und halten hier eine mehr circuläre Verlaufsweise ein, wobei sie Capillaren nur von rückwärts aufnehmen. Ein vollständig geschlossener terminaler Ring von venösen Anastomosen, wie er sich bei Wiederkäuern findet (Circulus venosus retinae anterior) kommt bei dem Menschen nicht vor. Eine gute und practische Schematisirung über Theilung und Ausbreitung der macroscopischen Gefässe der Retina des Menschen besitzen wir von H. Magnus*).

An dem Capillarnetz, welches aus der Verästelung der feinsten Gefässe der Retina hervorgeht, haben wir, wie an jedem andern, zu unterscheiden: den Durchmesser der Capillaren und die Ausdehnung, so wie die Gestalt der Maschen. Was den ersteren betrifft, so gehören die Retinacapillaren za den feinsten und können in dieser Beziehung nur mit jenen des Gehirns verglichen werden. Das Lumen derselben wechselt zwischen 4 und $6 \mu$. Auch die Maschen der Retinacapillaren ähneln sehr denen der grauen Gehirnsubstanz. Wie bei diesen ist die Gestalt vollkommen unregelmässig und eine gut ausgeführte Injection der Retina erinnert am meisten an die Geduldund Legspiele der Kinder. Es kann deshalb auch die Entfernung zweier Capillaren von einander, als Ausdruck der Grösse der Maschen, nicht genau bestimmt werden. Die Maasse wechseln hier zwischen $50-90 \mu$. Doch haben zahlreiche Messungen ergeben, dass die grosse Mehrzahl der Maschen einen Durchmesser von $60-75 \mu$ hat und dass der Längsdurchmesser mit dem Queruluichmesser nicht übereinstimmt, sondern bald der eine, bald

*) Die macroscopischen Gefässe der menschlichen Netzhant. Mit zwei lithogr. Taf. Leipzig, 1873, 
der andere länger ist. Uebrigens ist vücksichtlich der Gefässverhältnisse der Retinacapillaren immer in das A uge zu fassen, dass wir an unseren Injectionspräparaten der Retina nur die Anordnung der Gefässe in einer Ebene beobachten können, weshalb uns dabei nur zwei Durchmesser der Maschen entgegentreten. Allein das Retinagefässnetz hat eine dritte, die Tiefendimension, da die Capillaren, wie bereits oben erwähnt, bis zur äusseren Körnerschicht nachgewiesen werden können und demnach von der Zellenschicht durch die innere granulirto Lage und innere Körnerschicht sich erstrecken. Merï. dionale Durchschnitte der injicirten Retina geben abe ${ }^{r}$ nie ein Bild der Capillarmaschen, da die letzteren weiter sind als die Dicke microscopisch brauchbarer Schnitte and man daher an Schvitten der injicirten Retina woln Retinacapillaren, aber keine Capillarmaschen beobachten kann. Es ist daher mehr als wahrscheinlich, dass die oben geschilderten Formverhältnisse der Capillarmaschen der Retina wesentliche Modificationen erleiden, wenn dabei die dritte oder Tiefendimension der Retina berücksichtigt wird.

Ehe ich zur Beschreibung der Gefässverhältnisse der Umgebung des gelben Fleckes übergehe, glaube ich noch zweier Punkte gedenken zu mïssen, welche sich auf die Capillarität der ganzen Retina beziehen.' Zuerst kommt hier in Betracht die Länge des Capillarnetzes der Retina, das heisst die Entfernung der feinsten Arterienäste bis zu den Venenanfängen. Bei Durchmusterung verschiedener Stellen der Retina ergaben sich für diese Entfernung ziemlich constante Grössen. Die Länge der eigentlichen Capillarbahn beträgt nämlich selten weniger als $0,5 \mathrm{~mm}$ und goht auf der anderen Seite nur ausnahmsweise über $0,6 \mathrm{~mm}$. Nur innerhalb solcher kurzer Strecken fliesst das Blut in den eigentlichen Capillaren. Der zweite Punkt, welcher mir bei 
Beobachtung injicirter Retinapräparate auffiel, war der, dass in der unmittelbaren Umgebung der Arterien, bis zu den ganz feinen, ein Raum übrig bleibt, der frei von Capillaren ist, den wir demnach den capillarfreien Raum nennen wollen. An den Venen kommt ein solcher capillarfreier Raum nicht vor, was davon herrührt, dass selbst stärkere Retinavenen in grosser Anzahl unmittelbar Capillaren aufnehmen, die Venen in viel unmittelbarerer Verbindung mit den Capillaren stehen als die Arterien, deren terminale Ausläufer allein in das Capillarnetz übertreten. Nur ganz selten kommt es vor, dass eine wirkliche Retinacapillare seitlich mit einem als Arterie nachweisbaren Gefäss in Zusammenhang steht. Der Abstand des Capillarnetzes von der Arterienwand oder, was dasselbe ist, der Durchmesser des capillarfreien Raumes betrăgt $0,110-0,120 \mathrm{~mm}$. Vergl. Taf. IV. Fig. 1.

Der Arterien, welchen die Besorgung der den gelben Fleck umgebenden Capillarität obliegt, sind drei. Eine, welche über dem gelben Fleck, und eine andere, die unter demselben hinzieht. Diese beiden Arterien verlaufen etwas bogenförmig um die Macula lutea und sind unter dern Namen Arteriae maculares superior und inferior bekannt. $\mathrm{Zu}$ denselben gesellt sich als dritte ein kleineres arterielles Gefäss, welches nahe an der Papilla nervi optici von der Arteria centralis abgeht und sich dem medialen Pol der Macula lutea zuwendet, während der laterale Pol von den Endverästelungen der Arteriae superior und inferior versorgt wird. Die Entfernung der letzteren Arterienäste bis zum Rande der Micula lutea beträgt $0,5 \mathrm{~mm}$. Die Maschen des aus den Arterienverästelungen hervorgehenden Capillarnetzes zeichnen sich da, wo sie den gelben Fleek umgeben, von den übrigen Capillarmaschen der Retina dadurch aus, dass ihr Längsdurchmesser den Breitendurchmesser um fast 
mehr als das Doppelte übertrifft, das heisst, sie sind exquisit länglich und der Längsdurchmesser geht parallel mit dem Rande des gelben Fleckes. Dieses Capillarnetz ist nun durch den gelben Fleck scharf begrenzt und anch nicht eine Masche aberschreitet den Rand des letzteren. An dem mir vorliegenden Präparate tritt diese Thatsache um so evidenter hervor, als an demselben die Farbe des gelben Fleckes noch ziemlich gut erhalten ist. An Spirituspräparaten bleicht der gelbe Fleck ziemlich rasch aus. Unser Präparat wurde aber, bevor es mit Canadabalsam übergossen war, nicht seines Wassergehaltes durch Alkohol beraubt, sondern man liess die auf der Glasplatte ausgebreitete injicine Retina vor der Behandlung mit Canadabalsam einfach austrocknen, wodurch zwar die Strukturverhältnisse mit Ausnahme der Gefässanordnung in Verlust gingen, die Farbe der den gelben Fleck kennzeichnenden Stelle dagegen erhalten blieb. Dieselbe ist in der Mitte entschieden viel stärker ausgesprochen, als an den Rändern und verliext sich erst ganz an der Stelle, an welcher die Umsäumung durch das Capillarnetz beginnt.

Was die Venen betriff, welche das Blut aus dem den gelben Fleck umrandenden Capillarnetz abführen, so entsprechen dieselben nicht in ihrem Verlaufe den drei oben erwäbnten Arterien, sondern das einzige, was ich an diesen Venen beobachten konnte, ist dieses, dass sie im Allgemeinen eine in Beziehung auf den gelben Fleck radiäre Verlaufsweise einhalten.

Auf das mir vorliegende Präparat gestützt, wage ich den Ausspruch, dass der gelbe Fleck vollkommen gefässlos sei, und glaube zur Begründung desselben ausser der directen Beobachtung auch noch das schon oben erwähnte Gesetz rücksichtlich der Vertheilung der 
Blutgefässe in der Retina anführen zu können, nach welchem nur die vervösen Lagen der Retina gefässhaltig sind, in den musivischen dagegen die Blutgefüsse fehlen. Nun sind aber bekanntlich in dem gelben Fleck nur die letzteren vertreten und derselbe kann deshalb, wenn obiges Gesetz allgemeine Giltigleit hat, auf Blutgefässe keinen Anspruch machen. Was die ophthalmoscopischen Angaben betrifft, nach welchen in dem gelben Fleck Blutgefüsse beobachtet worden sein sollen, so glaube ich, dass man mittelst der Beobachtung durch den Augenspiegel, die überhaupt nur eine sehr geringe Vergrösserung zulâsst, Capillargefässe gar nicht sehen kann. Und was die oben erwähnten Angaben von Niemetschek und Nettleship betrifft, so können dieselben nur ihre Erklärung in unvollkommenen oder forcirten Injectionsversuchen finden, da unter diesen Verhältnissen allein der Anschein eines nicht völlig geschlossenen, den gelben Fleck umgejenden und dessen Rand überschreitenden Capillarnetzes denkbar ist.

Die anatomische Thatsache, dass hart an dem Rande des gelben Fleckes erst das Capillarnetz der Retina beginnt, giebt uns auch ein Mittel an die Hand, die Grösse des gelben Fleckes zu bestimmen. Wenn auch in dieser Beziehung individuelle Verschiedenheiten vorkommen, so war bisher doch nur eine approximative Grössenbestimmung des gelben Fleckes möglich, weil sich die gelbe Farbe allmählig in der Umgebung verliert. An dem mir vorliegenden Präparate, welches, wie oben erwähnt, der Retina eines vierjährigen Kindes entnommen ist, hatte der gelbe Fleck eine ganz exquisit ovale Gestalt, und zwar betrug der horizontale Durchmesser $1,02 \mathrm{~mm}$, während der verticale nur $0,92 \mathrm{~mm}$ mass. Ich bin weit entfernt, diese Zahlen als absolute für die Grösse des gelben Fleckes aufstellen zu wollen; jedenfalls sind sie 
aber die allein bis jetzt scharf bestimmten und werden so lange massgebende bleiben, bis in der Injectionstechnik gewandtere Anatomen eine grössere Anzahl von fehlerfreien Retinainjectionen des Menschen liefern werden, worüber bei der Schwierigkeit der Technik sowohl, wie bei der, passendes Material zu erhalten, noch einige Zeit hingehen dürfte.

Erklärung der Abbildunger.

Injicirte Retina mit Macula lutea von einem vierjahrigen Kinde.

Vergr. 25/1.

Fig. 1. A. Arterie.

V. Vene.

CFR. Capillarfreier Raum.

Fig. 2. Ams. Arteria macularis superior.

Ami. " " $\quad$ inferior.

Amm. " " media.

У. Vene.

MI. Macula lutea. 
Dafel 4

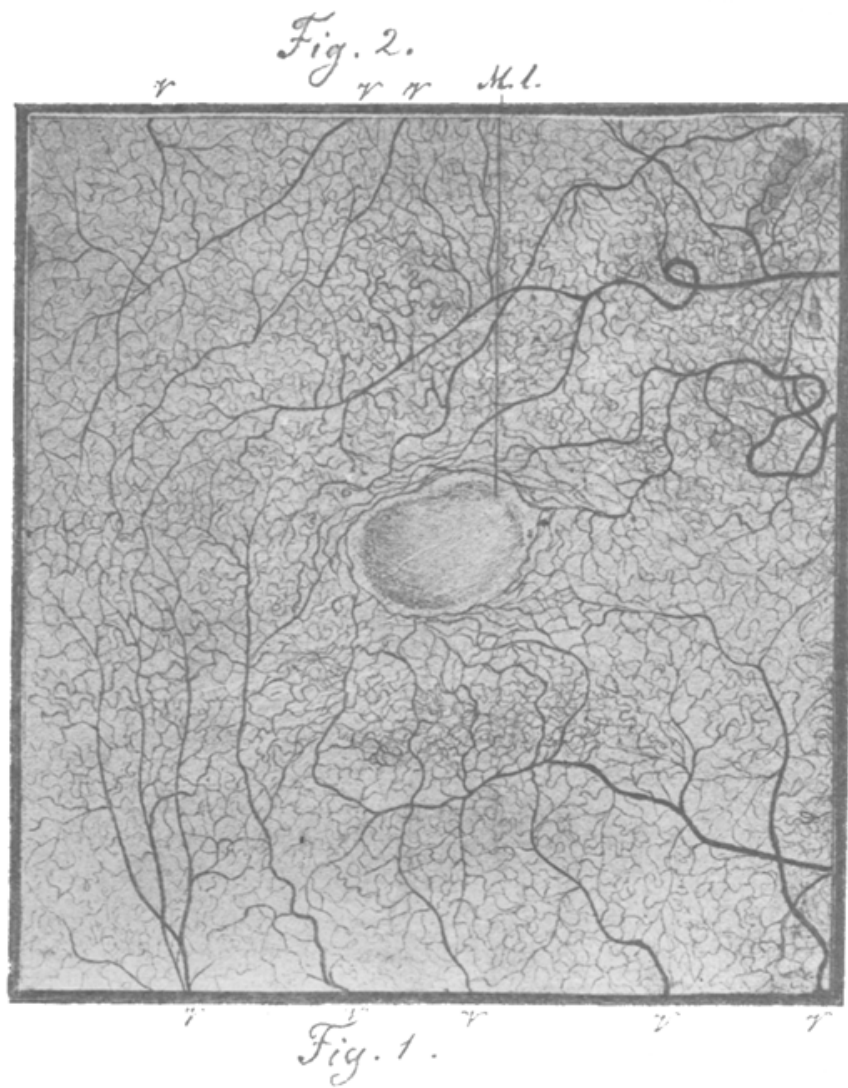

t.m.s.
A.m.m.

t.m.i.

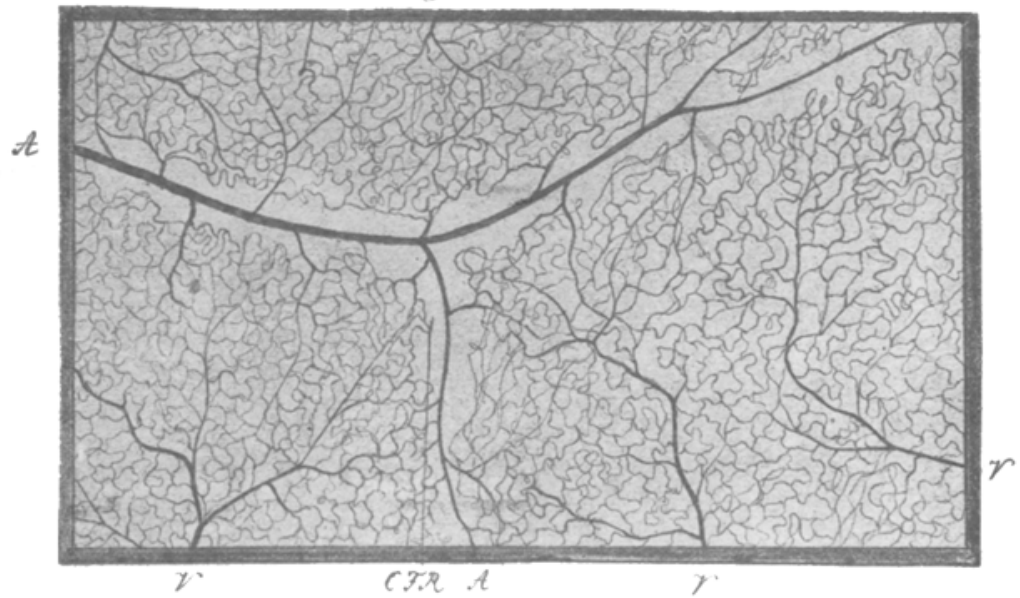

